\title{
Dibenazoazepine, A Pharmacologically Active Moiety
}

\author{
P. Panneerselvam ${ }^{1}$, Sandhya. $\mathrm{Kot}^{2}$, Vijaya lakshmi.K. ${ }^{3}$ \\ ${ }^{I}$ C.L.Baid Metha College of Pharmacy, Thoraipakkam, Chennai-600097
}

\begin{abstract}
The aim of this review was to collate literature work reported by many researchers on Dibenzazepine derivatives for their varoius pharmacological activities and also to report the recent efforts made on this moiety. This interesting group of compound has diverse biological activities such as antimicrobial, antimyobacterial, antiviral, analgesic, anticonvulsant, antidepressant, anticancer, antidiabetic, antioxidant. The purpose of this review is to provide an overview of diverse pharmacological activities of Dibenzazsepine. Various derivatives of Dibenzazepine have been analysed in this review for varying pharmacological activities.

Key Words: Dibenzazepine derivatives, antiviral, anticancer, anticonvulsant, antimicrobial, antidepressant .,
\end{abstract}

\section{Heterocyclic Chemistry: 1}

\section{Introduction}

Heterocyclic chemistry is the chemistry branch dealing exclusively with synthesis, properties and applications of heterocyclic. A heterocyclic compound is a cyclic compound which has atoms of at least two different elements such as $\mathrm{N}, \mathrm{S}$, and $\mathrm{O}$ as members of its ring(s).

\section{Azepines: 2}

Among 7 membered rings, azepine derivatives have been found to be associated with diverse pharmacological activities such as antiviral activity, anticancer activity, anticonvulsant activity, antidepressant activity, anti-insecticidal activity, vasopressin (AVP) antagonist activity. Among azepine derivatives, benzene fused rings having more biological activity.

Dibenzoazepine:3

Dibenzazepine (iminostilbene) is a chemical compound with two benzene rings fused to an azepine group fused to an azepine group. Dibenzazepine is used as an intermediate for the synthesis of specific analgesic and antipsychotic agents. Many anticonvulsants, tricyclic antidepressants (TCAs) including carbamazepine, oxcarbazepine, eslicarbazepine, clomipramine, desipramine, doxepin, imipramine, imipraminoxide, lofepramine, metapramine, opipramol, quinupramine, and trimipramine contain saturated dibenzazepine moieties in their chemical structures.

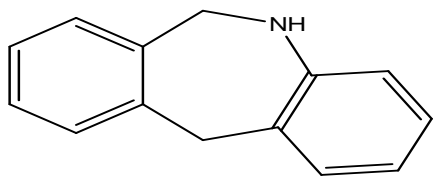

Naturally occurring Montanine, Coccinine, Manthine, Pancracine are the onlyalkaloids known to possess the morphanthridine (dibenzazepine) ring systempresentin Haemanthus and Rhodophiala species.

\section{Pharmacological Activities Based On Literature Review}

\section{Introduction:}

Epilepsy is a collective term for a group of chronic seizure disorders having in common, sudden and transient episodes (seizures of loss or disturbance of consciousness, usually but always with a characteristic body movement (convulsion) and sometimes with autonomic hyper activity ${ }^{85}$. The seizure nearly always correlates with an abnormal electrical discharge. The goal of an anticonvulsant is to suppress the rapid and excessive firing of neurons that start a seizure. Antiepileptic drugs function to help reduce this area of irritability and thus prevent epileptiform seizures ${ }^{86}$.

\section{Mechanism of action:}

Mechanism of action of the anticonvulsant agents in pain syndromes remains uncertain. Potential explanations include enhanced gamma-aminobutyric acid inhibition or a stabilization of the neuronal cell membranes or action via n-methyl-d-aspartate receptor sites. Carbamazepine, oxcarbazepine, lamotrigine and phenytoin act on voltage-gated sodium channels, while gabapentin and pregabalin act on voltage-gated calcium channels.topiramate and tiagabine potentiate gamma-aminobutyric acid inhibition. There is considerable variability in pain relief with the anticonvulsant agents. Some patients obtain relief with serum concentrations less than the therapeutic range in seizure disorders, whereas others require much higher dosing. Monitoring of serum drug concentrations is generally not necessary when dosing an anticonvulsant for pain or mood disorders. 
Epileptic seizure can be classified into,

1. Primarily generalized seizures

a. Grand-mal epilepsy or tonic-clonic seizure

b. Tonic seizure

c. Clonic seizure

d. Petit-mal epilepsy or absence seizure

f. Myoclonic seizure

2. Partial seizure

a. Simple partial seizure

b. Complex partial seizure

3. Secondarily generalized seizures or cortical focal epilepsy or jacksonian epilepsy.

\section{Mechanism of action:}

Mechanism of action of the anticonvulsant agents in pain syndromes remains uncertain. Potential explanations include enhanced gamma-aminobutyric acid inhibition or a stabilization of the neuronal cell membranes or action via n-methyl-d-aspartate receptor sites. Carbamazepine, oxcarbazepine, lamotrigine and phenytoin act on voltage-gated sodium channels, while gabapentin and pregabalin act on voltage-gated calcium channels.topiramate and tiagabine potentiate gamma-aminobutyric acid inhibition. There is considerable variability in pain relief with the anticonvulsant agents. Some patients obtain relief with serum concentrations less than the therapeutic range in seizure disorders, whereas others require much higher dosing. Monitoring of serum drug concentrations is generally not necessary when dosing an anticonvulsant for pain or mood disorders. Evaluation methods

The various methods used to evaluate anti-convulsant activity are as follows.

- Mes (maximal electrical shock) method

- Chemical (leptazol) method.

- Kindling method

- rota rod test

Drugs which antagonized convulsion induced by mes, leptazol and kindling are usually useful in grandmal. Petit-maland focal epilepsy respectively. Rota rod test are used to measure the effect of drug on their motor performance.

\subsubsection{Experimental method:}

Maximal electro-shock method in mice (mes) anti-convulsant activity of the synthesized compounds were screened by mes method. Phenytoin sodium at the dose of $25 \mathrm{mg} / \mathrm{kg}$ i.p. Was administered as std drugs for comparison. The test compounds at 2 dose levels of lowdose and high dose were administered i.p. The animals were held in suitable position and the corneal electrodes was placed on the cornea of the rat and apply $50 \mathrm{~m}$. A current for $0.2 \mathrm{sec}$ after half an hour administration of best compounds. Then the time spent by animal in each phase of convulsion was recorded. The reduction in time or abolition of tonic extensor phases was recorded. Animals in each extensor response was abolished were taken as protected rat.

$\%$ protection $=[($ control-test $) /$ control $] \times 100$ 
Effect of test molecules on seizure level in PTZ induced diabetic rats

\begin{tabular}{|c|c|c|c|c|c|}
\hline Groups & Treatment & Dose $(\mathrm{mg} / \mathrm{kg})$ & $\begin{array}{c}\text { Onset of } \\
\text { myoclonic } \\
\text { seizure } \\
(\mathrm{Sec})\end{array}$ & $\begin{array}{c}\text { Duration of } \\
\text { myoclonic } \\
\text { seizure } \\
(\text { Sec })\end{array}$ & $\begin{array}{c}\text { Seizure } \\
\text { Protection } \\
\%\end{array}$ \\
\hline 1. & $\begin{array}{c}\text { Normal } \\
\text { Saline }\end{array}$ & $10 \mathrm{ml}$ & $124.33 \pm 1.45$ & $135.00 \pm 2.517$ & 0 \\
\hline 2. & $\begin{array}{c}\text { Standard } \\
\text { Diazepam }\end{array}$ & 5 & $299.66 \pm 0.33$ & $5 \pm 0.57$ & 100 \\
\hline 3. & D1 & 200 & $195.26 \pm 1.45$ & $56 \pm 1.35$ & 56.15 \\
\hline 4. & D2 & 200 & $253.00 \pm 0.51$ & $24 \pm 0.52$ & 80.69 \\
\hline 5. & D3 & 200 & $162.86 \pm 0.84$ & $70.96 \pm 0.84$ & 46.72 \\
\hline 6. & D4 & 200 & $174.33 \pm 0.82$ & $64.53 \pm 0.73$ & 51.58 \\
\hline 7. & D5 & 200 & $231.62 \pm 1.201$ & $36 \pm 1.51$ & 72.24 \\
\hline 8. & D6 & 200 & $157.31 \pm 1.44$ & $76.31 \pm 0.82$ & 43.5 \\
\hline 9. & D7 & 200 & $223.56 \pm 1.15$ & $42 \pm 0.55$ & 68.73 \\
\hline 10. & D8 & 200 & $246.33 \pm 1.74$ & $31 \pm 0.59$ & 77.71 \\
\hline 11. & D9 & 200 & $211.09 \pm 0.61$ & $44.98 \pm 0.29$ & 79.42 \\
\hline 12. & D10 & 200 & $204 \pm 0.19$ & $52 \pm 1.51$ & 63.12 \\
\hline 13. & D11 & 200 & $265.33 \pm 1.453$ & $22.66 \pm 0.88$ & 96.1 \\
\hline 14. & D12 & 200 & $179.66 \pm 1.73$ & $61.66 \pm 0.85$ & 53.82 \\
\hline
\end{tabular}

The values are expressed as Mean \pm SEM, $n=3$

Effect of test molecules on seizure level in PTZ induced diabetic rats

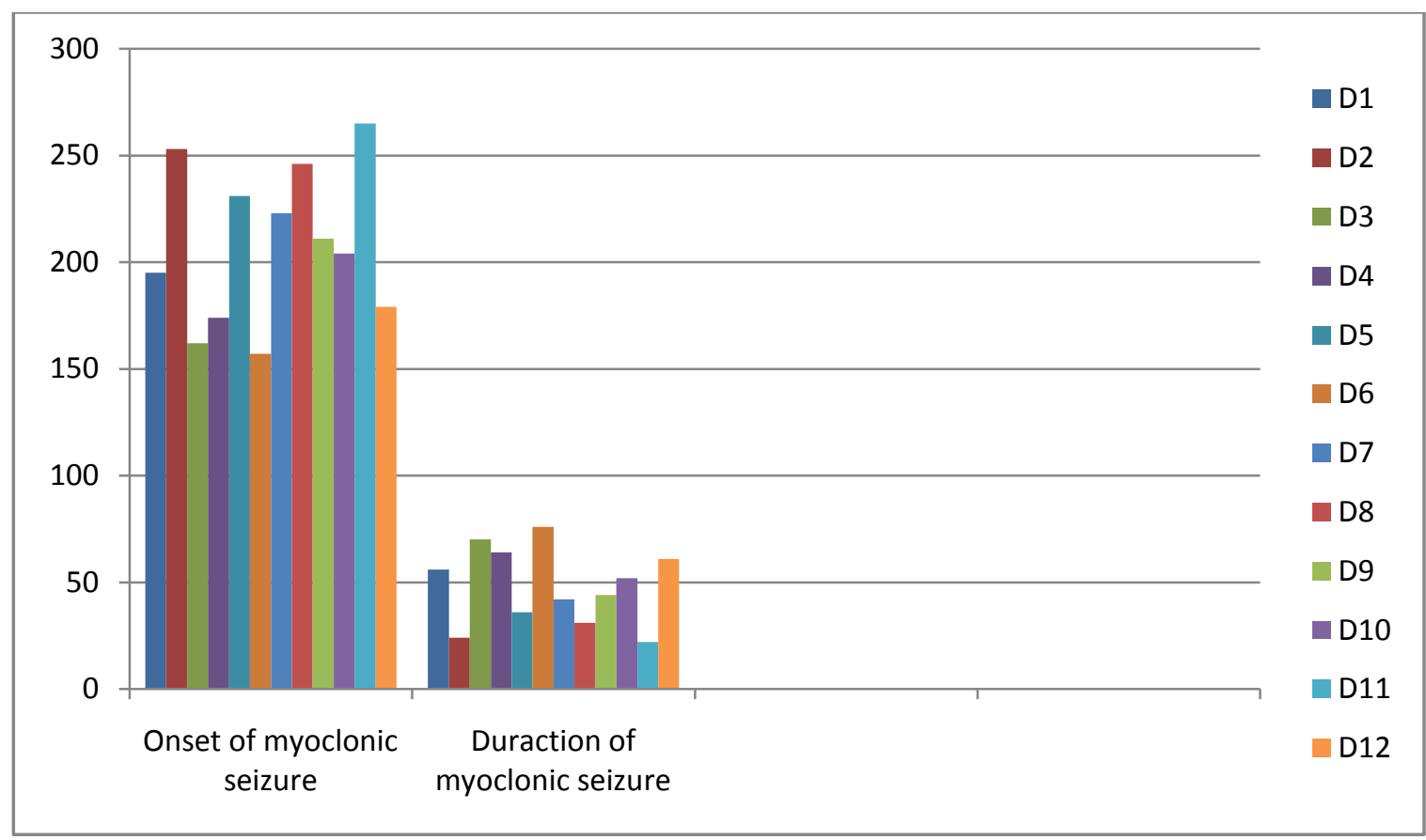




\section{Results and discussion:}

- Method was used to study the anti-convulsant activity of the synthesized compounds when compared with (Diazepam $5 \mathrm{mg} / \mathrm{kg}$ i.p) as standard drug.

- The study was carried at one dose level $(200 \mathrm{mg} / \mathrm{kg}, \mathrm{p} . \mathrm{o})$ and the effect of drug on tonic flexion and tonic extensor phase was recorded.

- $\quad$ all the compounds exhibited significant anti-convulsant activity

- All compounds exhibited more action on extensor phase than flexion phase.

- The present study, all the test molecules showed mild to good anticonvulsant activity in the dose of $200 \mathrm{mg} / \mathrm{kg}$ and compared with standard drug.In addition graded dose respons was also observed.

from the above results it may concluded that compounds containing electron donating groups exhibits better activity than electron withdrawing groups.

\section{Reference:}

[1] Naveen vasudev kulkarni, gurunath suresh kurdekar, “ anticonvulsant activity and toxicity evaluation of dibenazoazepine.

[2] m. Veverka, chem. Papers 1992, 46(3), 206_210.[15] p. A. Wolf, w. M. Westveer, arch. Biochem, 1950, 28,201_206.

[3] t. Kotani, i. Ichimoto, c. Tatsumi, t. Fujita, agr. Biol. Chem. 1975, 39(6), 1311_1317.

[4] m. Uher, v. Konecny, o. Rajniakova, chem. Papers 1994, 48(4), 282_284.

[5] m. Yamato, y. Yasumoto, j

[6 ]Maryam Shekarchia, Marya Binesh Marvastia, Mohammad Sharifzadehb and Abbas Shafieea. Anticonvulsant Activities.

[7] Abdulrahman M. Al-Obaid, Hussein I. El-Subbagh, Othman A. Al-Shabanah and Mohamed M. Elmazar. Synthesis and Anticonvulsant Activity.

[ 